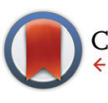

CrossMark

\& click for updates

Cite this: Dalton Trans., 2016, 45 2116

Received 20th August 2015 Accepted 9th October 2015

DOI: $10.1039 / c 5 d t 03226 a$

www.rsc.org/dalton

\title{
Modular solid-phase synthesis, catalytic application and efficient recycling of supported phosphine-phosphite ligand libraries $\uparrow$
}

\author{
Frank J. L. Heutz and Paul C. J. Kamer* \\ In spite of decades of research in the field of homogeneous asymmetric catalysis the discovery of new \\ high performance catalysts still relies heavily on trial-and-error. There is still a lack of efficient combina- \\ torial methods which enable the synthesis and screening of vast ligand libraries, especially for bidentate \\ phosphorus ligands. Here we present a highly modular solid-phase synthetic approach which provides \\ facile access to libraries of phosphine-phosphite ligands in quantitative yield requiring only minimal \\ work-up. The obtained library of supported phosphine-phosphites was successfully applied in rhodium \\ catalyzed asymmetric hydrogenation obtaining high enantioselectivities up to $98 \%$. Also, these polymer \\ supported ligands could be successfully recycled under batch conditions exhibiting only a small decline \\ of activity and no loss of selectivity.
}

\section{Introduction}

Over the last few decades transition metal mediated asymmetric catalysis has evolved into an indispensable tool to both academia and industry, providing access to optically active compounds such as flavors and fragrances, pharmaceuticals and agrochemicals. ${ }^{1}$ Despite the fact that nowadays homogeneous asymmetric catalysis is a highly evolved field of research, the discovery of high performance ligands for asymmetric transformations remains challenging. ${ }^{2}$ Although our knowledge of organometallic chemistry and ligand effects in catalysis is ever increasing and computational techniques play an increasingly important role, ${ }^{3}$ the development of new catalysts still relies heavily on trial-and-error. ${ }^{4}$

Combinatorial synthetic methodologies and high-throughput screening have proven their value in asymmetric catalysis and numerous successful approaches have been reported using both covalent ${ }^{5}$ and supramolecular chemistry. ${ }^{6}$ For phosphorus donor ligands the focus has mainly been on standard solution-phase techniques and mainly monodentate ligands. ${ }^{7}$ Large libraries of bidentate phosphorus ligands and efficient combinatorial methods for synthesizing these remain scarce due to their intrinsically more complicated synthesis and work-up procedures. ${ }^{8}$ This is even more the case for heterobidentate phosphorus ligands like phosphine-phosphites,

EaStCHEM, School of Chemistry, University of St Andrews, North Haugh, St Andrews KY16 9ST, UK. E-mail: pcjk@st-andrews.ac.uk

$\dagger$ Electronic supplementary information (ESI) available: Experimental and characterisation data. See DOI: 10.1039/c5dt03226a which can be attributed to the difficulty of introducing two different phosphorus moieties onto the ligand backbone.

Heterobidentate P-OP ligands such as phosphine-phosphites have received increased attention over the last years and have proven to be very efficient ligands for various asymmetric transformations such as hydrogenation, hydroformylation and allylic substitution. ${ }^{9}$ BINAPHOS, first reported by the group of Takaya and Nozaki, ${ }^{10}$ has been highly effective in a wide range of reactions and belongs to the small group of so-called privileged ligands in asymmetric catalysis. ${ }^{2}$ The highly modular structure of phosphine-phosphites and the fact they possess two electronically different phosphorus moieties, resulting in different trans-labilizing properties, makes them promising candidates for the combinatorial synthesis and high-throughput screening of structurally diverse ligand libraries. ${ }^{11}$ There have been few reports of modular approaches towards phosphine-phosphite ligand but so far the applications remain fairly limited. ${ }^{12}$ The preparation of this class of ligands still relies on classical solution-phase methodologies and work-up procedures and suffers from relatively low yields, thus hampering truly high-throughput synthesis and screening.

Solid-phase synthesis (SPS) provides a promising alternative approach towards ligand libraries and has already successfully been employed for many years for the synthesis of large compound libraries such as polypeptides and oligosaccharides. ${ }^{13}$ Using solid-phase synthesis, ligand structures can be built up step-by-step in a combinatorial fashion while being bound to a resin bead. Using a solid support has as main advantage that it can greatly simplify ligand purification procedures which in turn allows the use of large excesses of reagents to drive 
reactions to completion. Often the work-up only comprises a simple filtration or decantation step as opposed to the laborious work-up procedures employed in the solution-phase synthesis of phosphorus ligands. ${ }^{14}$ The facile work-up makes solid-phase synthesis very suitable for automated synthesis using high throughput equipment.

An additional advantage of employing a solid support is that it can greatly facilitate catalyst recovery after the catalytic reaction and potentially even lead to recyclable immobilized catalysts. There are numerous accounts of immobilized (chiral) phosphorus ligands addressing catalyst recovery, which presents one of the major problems in homogeneous catalysis. The vast majority however, are reports of nonmodular single ligands or catalysts anchored to a support. ${ }^{15}$

Surprisingly, the application of solid-phase synthesis for the combinatorial synthesis of supported bidentate phosphorus ligands remains fairly limited. ${ }^{8}$ However, there have been reports on SPS of a small variety of aminophosphinephosphine and aminophosphine-phosphite libraries ${ }^{16}$ and recently we reported on the solid-phase synthesis of diphosphine ligands. ${ }^{17}$ Using a similar methodology we now report on an efficient combinatorial solid-phase synthetic approach for libraries of highly modular and recyclable phosphinephosphite ligands.

\section{Results and discussion}

\section{Solid-phase synthesis of phosphine-phosphite ligands}

A ligand library of 16 supported phosphine-phosphite (P-OP) ligands was prepared of which the general structure is depicted in Fig. 1. These ligands possess a highly modular structure and by varying the three main building blocks in a combinatorial fashion it is possible to quickly generate a ligand library showing large structural diversity. The phosphine moiety can be altered by using phosphines bearing different substituents $\left(R^{1}\right)$ and for the ligand backbone cyclic sulfates with a varying backbone length $(n)$ and different substituents $\left(R^{2}\right)$ can be used. Finally diversity in the -OP part of the ligand can be created by employing various chlorophosphites to introduce the phosphite moiety giving in total 4 points of diversity.

We have developed a modular stepwise methodology for the synthesis of P-OP ligands (see Scheme 1). The initial synthetic steps for the preparation of the intermediate supported phosphine-borane sulfates are identical to those reported for
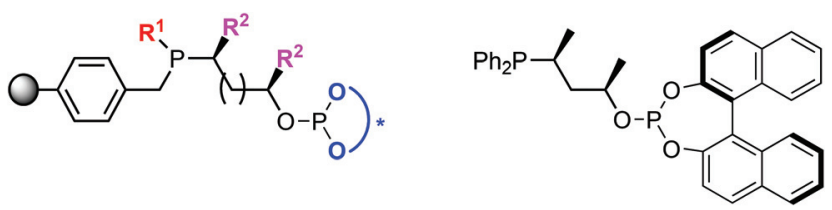

Fig. 1 General structure of highly modular supported phosphinephosphite ligands (left), and a solution-phase analog reported by Bakos et al. ( $L_{17}$, right). ${ }^{18}$ supported diphosphines. ${ }^{17}$ Subsequently, the sulfate group is hydrolyzed and the resulting hydroxyalkyl phosphines are reacted with chlorophosphite reagents yielding supported phosphine-phosphite ligands. A similar solution-phase procedure has been reported by the group of Bakos and one of their homogeneous analogues of our supported P-OP ligands is depicted in Fig. $1 .^{18}$

Using our solid-phase synthetic approach this class of ligands is readily accessible under very mild conditions requiring only a very minimal work-up procedure between each reaction step. Moreover, the supported ligands were obtained in high purity with each reaction step proceeding quantitatively as determined by gel-phase ${ }^{31} \mathrm{P}$ NMR (see Fig. 2). Comparatively, when employing traditional solution-phase methodologies laborious purification methods like column chromatography and distillations under inert conditions are often required and the overall yield for similar phosphinephosphites can be as low as $30 \%{ }^{19}$

The starting synthon of the solid-phase synthesis are supported phosphine-boranes $\mathbf{1} \mathbf{a}-\mathbf{b} \cdot \mathbf{B} \mathbf{H}_{\mathbf{3}}$ which are readily accessible by treating a choromethyl functionalized resin, in this case Merrifield resin, with various primary lithium phosphides having different substituents $\left(R^{1}\right)$. The introduction of the phosphine moiety takes place in a non-stereospecific fashion and yields a racemic mixture. Subsequently the obtained supported secondary phosphines can be protected by treatment with $\mathrm{BH}_{3} \cdot \mathrm{SMe}_{2}$ to make them less prone to oxidation. $\mathrm{BH}_{3} \cdot \mathrm{SMe}_{2}$ was chosen as reagent over the THF adduct due to its higher stability and solubility. Upon deprotonation using lithium diisopropylamide (LDA, step I) the lithiated phosphine-boranes $\left(\mathbf{L i} \cdot \mathbf{1} \mathbf{a}-\mathbf{b} \cdot \mathbf{B} \mathbf{H}_{\mathbf{3}}\right)$ can be reacted with a cyclic sulfate which after ring opening, with full inversion at one of the stereogenic centers, ${ }^{20}$ serves as the ligand backbone (step II). Stronger bases such as $n$-butyl lithium resulted in side products due to transmetallation at the benzylic position. Diversity can be introduced by employing various cyclic sulfates having a varying backbone length (n) and bearing different substituents $\left(R^{2}\right)$. All the above mentioned reaction steps could be readily followed by ${ }^{31} \mathrm{P}$ NMR confirming the formation of the desired intermediates (see Fig. 2 for a representative example).

Next step is the hydrolysis of the supported phosphineborane sulfates $\left(\mathbf{3} \mathbf{a}-\mathbf{h} \cdot \mathbf{B} \mathbf{H}_{3}\right)$ to the corresponding hydroxyalkyl phosphines $\left(\mathbf{4} \mathbf{a}-\mathbf{h} \cdot \mathbf{B} \mathbf{H}_{3}\right)$. Bakos et al. have reported an analogous homogeneous procedure ${ }^{18}$ but their conditions, $90{ }^{\circ} \mathrm{C}$ using $2 \mathrm{M}$ sulfuric acid, were found to be too harsh and led to decomposition of the resin. Instead a very mild hydrolysis at room temperature was applied, using a 1:1 mixture of THF and $0.1 \mathrm{M}$ sulfuric acid to ensure proper swelling of the resin and to retain its structural integrity. The hydrolysis proceeded relatively slow and proved difficult to monitor by ${ }^{31} \mathrm{P} \mathrm{NMR}$ as there was no notable NMR shift observable, although the hydrolysis products did exhibit slightly sharper peaks (Fig. 2). Using ${ }^{7} \mathrm{Li}$ NMR however, it was possible to follow the hydrolysis in time. The consumption of the lithium sulfate group, exhibiting a peak around $1 \mathrm{ppm}$, could be readily monitored 


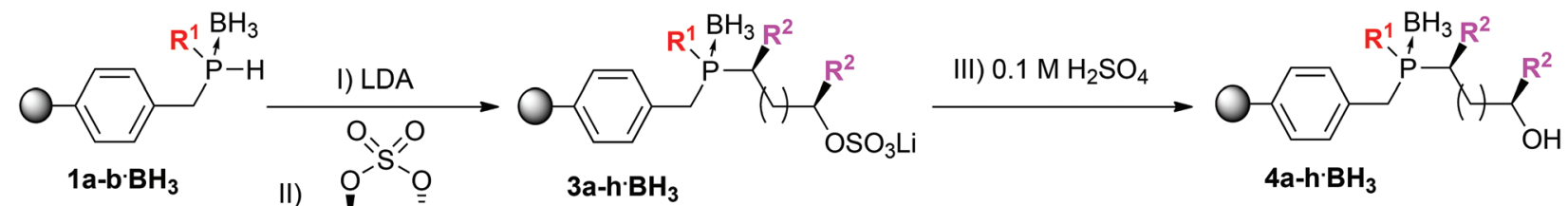<smiles>[R]C(C)C([R])C(C)C(C)C</smiles>

2a: $R^{2}=M e, n=1,\left(S_{C}, S_{C}\right)$

2b: $R^{2}=M e, n=1,\left(R_{\mathrm{C}}, R_{\mathrm{C}}\right)$

2c: $R^{2}=M e, n=2,\left(S_{C}, S_{C}\right)$

2d: $\mathrm{R}^{2}=\mathrm{Me}, \mathrm{n}=2,\left(R_{\mathrm{C}}, R_{\mathrm{C}}\right)$

2e: $R^{2}=\mathrm{Et}, \mathrm{n}=2,\left(R_{\mathrm{C}}, R_{\mathrm{C}}\right)$
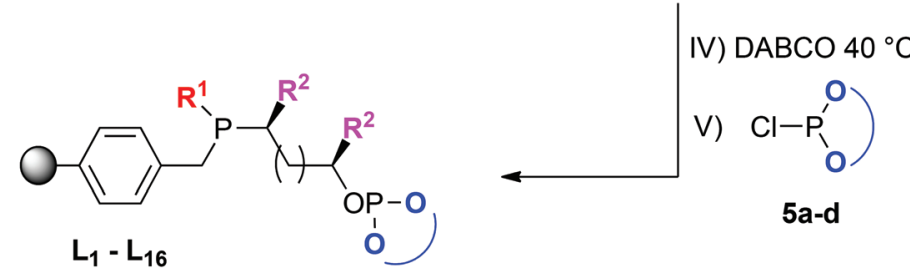

Scheme 1 Solid-phase synthetic approach towards supported phosphine-phosphite ligands, all reactions were performed in THF at room temperature unless stated otherwise.

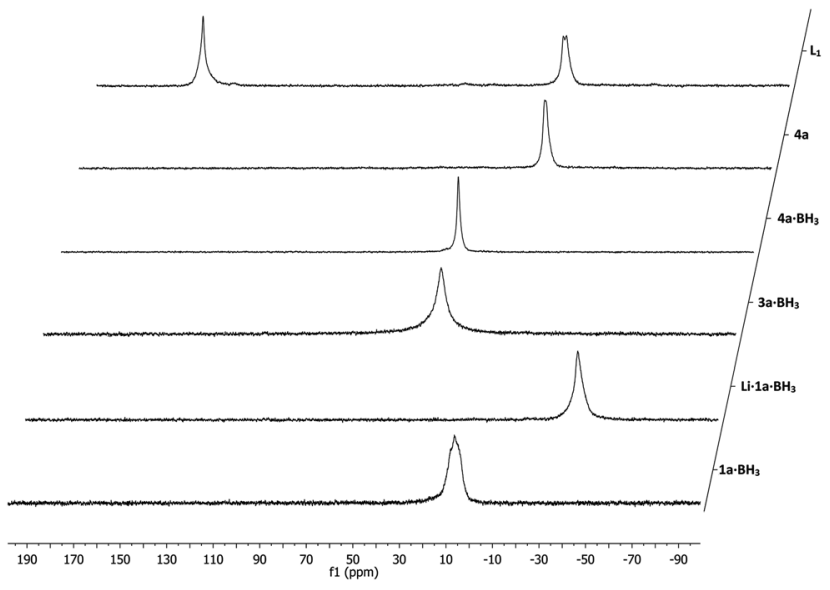

Fig. 2 Solid-phase synthesis of representative supported phosphinephosphite $L_{1}$ as monitored by gel-phase ${ }^{31} \mathrm{P}$ NMR spectroscopy.

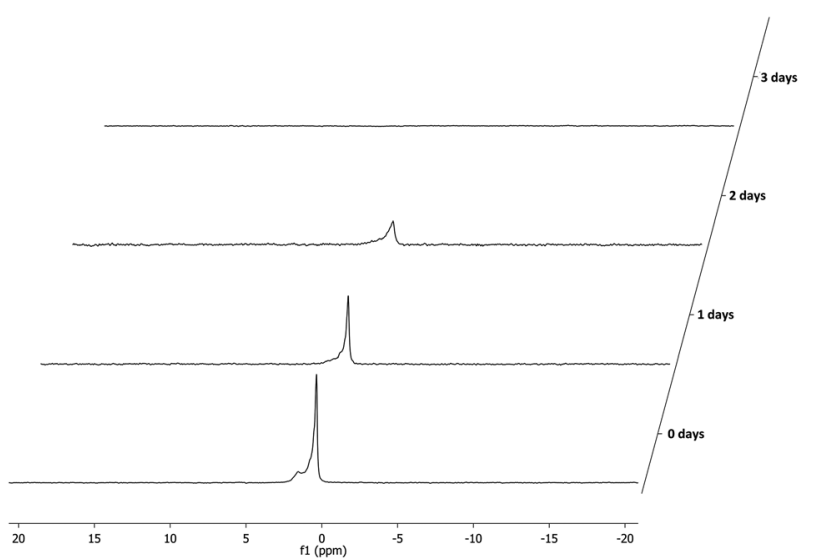

Fig. 3 Hydrolysis of representative supported phosphine-borane sulfate $3 \mathrm{a} \cdot \mathrm{BH}_{3}$ monitored by ${ }^{7} \mathrm{Li}$ NMR spectroscopy.

as seen in Fig. 3. Full hydrolysis was on average observed after 3 days and could also be confirmed using FT-IR and elemental analysis, both showing full removal of the sulfate ester group.
It was decided to perform the removal of the $\mathrm{BH}_{3}$ group prior to introduction of the -OP moiety as phosphites are known to be more prone to hydrolysis under basic conditions. ${ }^{21}$ The borane group was removed by treatment with an excess of 1,4-diazabicyclo[2.2.2] octane (DABCO, 10 eq.) at $40{ }^{\circ} \mathrm{C}$ which could be readily followed by ${ }^{31} \mathrm{P}$ NMR (Fig. 2). Next the phosphite moieties were introduced by treating the supported hydroxyalkyl phosphines $(\mathbf{4 a}-\mathbf{h})$ with various chlorophosphites in the presence of triethylamine. Both $(S)$ binol and $(R)$-binol derived chlorophosphites were employed as well as a slightly more bulky trimethylsilyl functionalized binol-PCl. Moreover, also a $t$-butyl functionalized bisphenol derived chlorophosphite was used, demonstrating the versatility of this solid-phase synthetic approach. In all cases the introduction of the -OP moiety proceeded smoothly although for the more bulky phosphites a slightly larger excess, up to 3 equivalents of reagent, was required.

Fig. 2 shows that this reaction step could be readily monitored using ${ }^{31} \mathrm{P}$ NMR and the appearance of a second peak in a $1: 1$ ratio could be observed confirming the formation of the desired supported P-OP ligands in high purity. In some cases, like for ligand $\mathbf{L}_{\mathbf{8}}$, splitting into two 1 : 1 signals for the phosphine or phosphite group could be observed (Fig. 4). This is due to the fact that all supported ligands are present as a mixture of two epimers at the phosphine moiety. But for most ligands only single broad peaks could be observed, probably

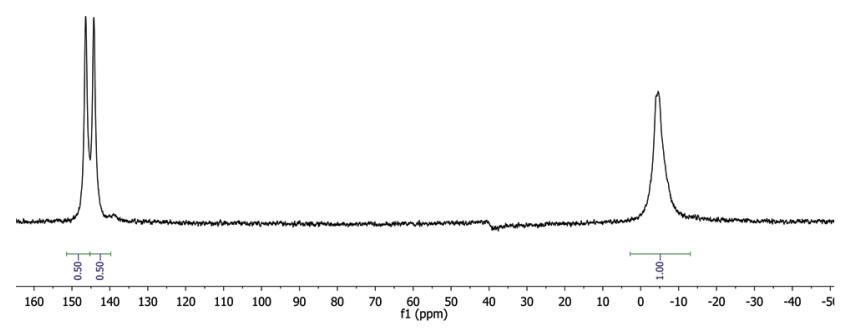

Fig. $4{ }^{31}$ P NMR spectrum of supported phosphine-phosphite $L_{8}$ clearly showing the presence of both epimers in a 1:1 ratio. 


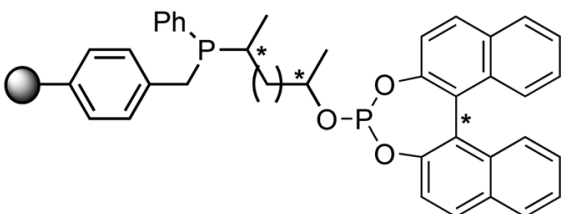

$\mathrm{L}_{1}: \mathrm{n}=1,\left(R_{\mathrm{C}}, \mathrm{S}_{\mathrm{C}}, \mathrm{S}_{\mathrm{ax}}\right)$

$\mathbf{L}_{2}: \mathbf{n}=1,\left(S_{C}, R_{C}, S_{\text {ax }}\right)$

$\mathbf{L}_{3}: \mathbf{n}=1,\left(R_{\mathrm{C}}, S_{\mathrm{C}}, R_{\mathrm{ax}}\right)$

$\mathrm{L}_{4}: \mathrm{n}=1,\left(S_{\mathrm{C}}, R_{\mathrm{C}}, R_{\mathrm{ax}}\right)$

$\mathrm{L}_{5}: \mathrm{n}=2,\left(R_{\mathrm{C}}, S_{\mathrm{C}}, S_{\mathrm{ax}}\right)$

$\mathrm{L}_{6}: \mathrm{n}=2,\left(\mathrm{~S}_{\mathrm{C}}, R_{\mathrm{C}}, S_{\mathrm{ax}}\right)$

$\mathrm{L}_{7}: \mathrm{n}=2,\left(R_{\mathrm{C}}, S_{\mathrm{C}}, R_{\mathrm{ax}}\right)$

$\mathrm{L}_{8}: \mathrm{n}=2,\left(S_{\mathrm{C}}, R_{\mathrm{C}}, R_{\mathrm{ax}}\right)$

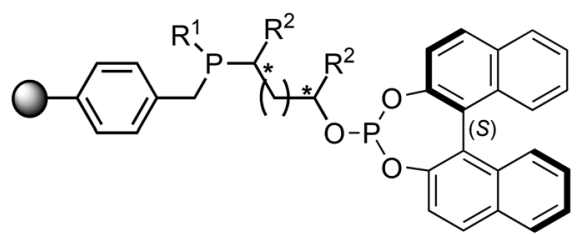

$$
\begin{aligned}
& \mathrm{L}_{g}: \mathrm{R}^{1}=\mathrm{Ph}, \mathrm{R}^{2}=\mathrm{Et}, \mathrm{n}=2,\left(S_{\mathrm{C}}, R_{\mathrm{C}}\right) \\
& \mathrm{L}_{10}: \mathrm{R}^{1}=\mathrm{Cy}, \mathrm{R}^{2}=\mathrm{Me}, \mathrm{n}=1,\left(R_{\mathrm{C}}, S_{\mathrm{C}}\right) \\
& \mathrm{L}_{11}: \mathrm{R}^{1}=\mathrm{Cy}, \mathrm{R}^{2}=\mathrm{Me}, \mathrm{n}=2,\left(R_{\mathrm{C}}, S_{\mathrm{C}}\right) \\
& \mathrm{L}_{12}: \mathrm{R}^{1}=\mathrm{Cy}, \mathrm{R}^{2}=\mathrm{Et}, \mathrm{n}=2,\left(S_{\mathrm{C}}, R_{\mathrm{C}}\right)
\end{aligned}
$$
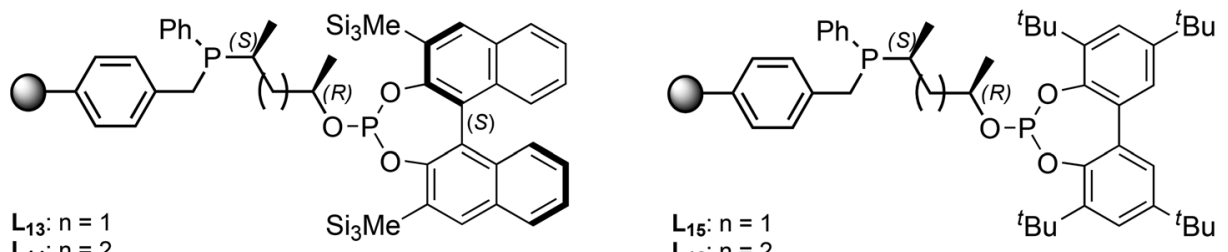

$L_{16}: n=2$

Fig. 5 Complete Library of supported phosphine-phosphite ligands $\left(L_{1}-L_{16}\right)$.

due to overlap of the two epimer signals. Deerenberg et al. reported that for similar phosphine-phosphite systems the P-stereogenic center has little influence on the chiral induction and that the selectivity is mainly determined by the ligand backbone and the phosphite moiety. ${ }^{22}$ Finally the actual phosphorus loading of the immobilized phosphinephosphites could be determined by elemental analysis. ${ }^{1} \mathrm{H}$ and ${ }^{13} \mathrm{C}$ NMR of the supported ligands was not very informative due very broad peaks and overlap with signals of the Merrifield resin (see ESI $\dagger$ for representative examples). The complete P-OP ligand library $\left(\mathbf{L}_{\mathbf{1}}-\mathbf{L}_{\mathbf{1 6}}\right)$ synthesized using this efficient solidphase synthetic approach is depicted in Fig. 5.

\section{Rh-catalyzed asymmetric hydrogenation}

The library of 16 supported phosphine-phosphite ligands was employed in the asymmetric hydrogenation of several benchmark substrates. The complexation was performed prior to catalysis by suspending the resin-bound ligands in dichloromethane in the presence of 0.9 eq. of rhodium precursor $\left(\left[\mathrm{Rh}(\mathrm{COD})_{2}\right] \mathrm{X}\right)$. After one hour the white resins had turned bright yellow/orange and the now colorless supernatant was removed. Subsequently the supported rhodium complexes were washed with DCM and THF and filtered off. To confirm full complexation, separate in situ NMR experiments were performed which indicated the expected bidentate coordination to the metal center. Upon complexation very broad NMR signals were observed, this phenomenon was already reported for similar resin-supported systems. ${ }^{17,23}$

Table 1 shows the results for the asymmetric hydrogenation of substrates I-III using supported ligands $\mathbf{L}_{\mathbf{1}}-\mathbf{L}_{\mathbf{8}}$. These 8 ligands all bear the same substituents $\left(R^{1}\right.$ and $\left.R^{2}\right)$ and differ only in the number of carbon atoms in the ligand backbone $(n)$ and the configuration of the backbone and phosphite moiety. In all cases full conversion was achieved and
Table 1 Results of Rh-catalyzed asymmetric hydrogenation using $\mathrm{L}_{1}-\mathrm{L}_{8}{ }^{a}$

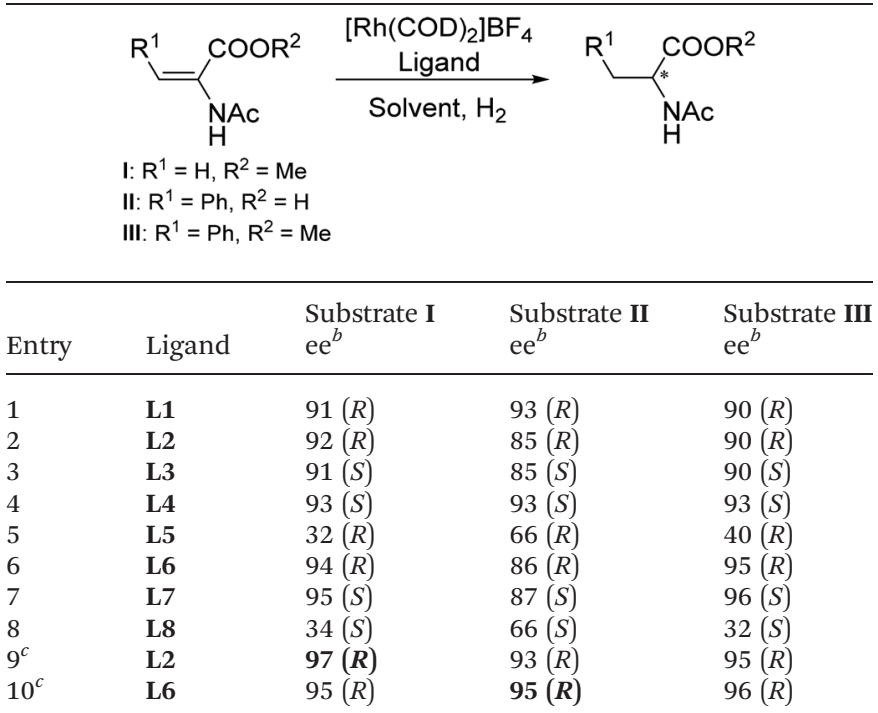

${ }^{a}$ Reaction conditions: In a stainless steel autoclave, $\mathrm{Rh} / \mathrm{substrate}=$ $1: 30, p\left(\mathrm{H}_{2}\right)=1.2 \mathrm{bar}, T=25^{\circ} \mathrm{C}, t=16 \mathrm{~h}, 0.5 \mathrm{~mL}$ of THF, all runs were performed in duplicate, full conversion was obtained in all cases, conversion was determined by GC. ${ }^{b}$ Enantiomeric excess of product determined by chiral GC (absolute configuration drawn in parenthesis). ${ }^{c}$ Using $\left[\mathrm{Rh}(\mathrm{COD})_{2}\right] \mathrm{SbF}_{6}$ as metal precursor.

enantioselectivities up to $97 \%$ were observed. Interestingly it was found that changing the counterion of the Rh-precursor from $\mathrm{BF}_{4}{ }^{-}$to $\mathrm{SbF}_{6}{ }^{-}$(entries 9 and 10) seemed to have a beneficial effect and led to an increase of ee of up to $9 \%$.This counterion effect has already been reported for similar homogeneous phosphite- and diamidophosphite-based systems. ${ }^{24}$ Looking at the ligand backbone length it can be seen that for substrate II higher enantioselectivities were obtained for 
ligands having a C3 backbone, $n=1,\left(\mathbf{L}_{\mathbf{1}}\right.$ and $\mathbf{L}_{\mathbf{4}}$, entries 1 and 4$)$ while for substrates I and III better selectivities were observed with supported phosphine-phosphites having a $\mathrm{C} 4$ backbone, $n=2,\left(\mathbf{L}_{6}-\mathbf{L}_{7}\right.$, entries $\left.6-7\right)$.

Also the influence of the configuration of the backbone and phosphite moiety was investigated for $\mathbf{L}_{\mathbf{1}}-\mathbf{L}_{\mathbf{8}}$. The $(S)$-Binol moiety always leads to the $(R)$ enantiomer and an $(R)$-Binol group yields the $(S)$ product in the asymmetric hydrogenation of substrates I-III (see Table 1). For the supported ligands bearing a $\mathrm{C} 3$ backbone $\left(n=1, \mathbf{L}_{\mathbf{1}}-\mathbf{L}_{\mathbf{4}}\right)$ a small matched/ mismatched effect could be observed with the matched pairs $\left(R_{\mathrm{C}}, S_{\mathrm{C}}, S_{\mathrm{ax}}\right)$ and $\left(S_{\mathrm{C}}, R_{\mathrm{C}}, R_{\mathrm{ax}}\right)$ achieving up to $8 \%$ higher selectivities in the case of substrate II (entries 1 and 4). Interestingly, for $\mathbf{L}_{\mathbf{5}}-\mathbf{L}_{\mathbf{8}}(n=2)$ a much larger and inverse matched $/$ mismatched effect was observed. In this case the matched pairs are $\left(R_{\mathrm{C}}, S_{\mathrm{C}}, R_{\mathrm{ax}}\right)$ and $\left(S_{\mathrm{C}}, R_{\mathrm{C}}, S_{\mathrm{ax}}\right)$ (entries 6 and 7$)$ which exhibited differences in enantioselectivity up to $62 \%$ when compared to their mismatched counterparts (entries 5 and 8).

Moreover, the effect of altering the substituents $R^{1}$ and $R^{2}$ was studied (Table 2, entries 1-4). It was found that replacing the methyl group on the ligand backbone $\left(R^{2}\right)$ with a slightly more bulky ethyl group appeared to have a small positive effect on the selectivity. When comparing $\mathbf{L}_{\mathbf{6}}$ (Table 1 , entry 6) with $\mathbf{L}_{\mathbf{9}}$ (Table 2, entry 1) it can be seen that the latter one bearing an ethyl group achieves up to $5 \%$ higher enantioselectivities. Exchanging the phenyl substituent on the phosphine moiety $\left(R^{1}\right)$ for a cyclohexyl group on the other hand seemed to have a detrimental effect. While $\mathbf{L}_{\mathbf{1 0}}$ (Table 2, entry 2) exhibits similar selectivity in the hydrogenation of $\mathbf{I}$ as its phenyl bearing counterpart $\mathbf{L}_{\mathbf{1}}$ (Table 1, entry 1) the selectivity for the other two substrates is up to $26 \%$ lower. Similarly, the enantioselectivities achieved by $\mathbf{L}_{\mathbf{1 2}}$ (Table 2, entry 4 ) are comparable to those of its phenyl-bearing counterpart $\mathbf{L}_{\mathbf{9}}$ (Table 2, entry 1 ) for two of the three substrates but for substrate II the selectivity is much lower. More surprisingly, when comparing $\mathbf{L}_{\mathbf{1 1}}$ (Table 2 entry 3 ) and $\mathbf{L}_{\mathbf{5}}$ (Table 1 , entry 5 ) it was observed that

Table 2 Results of Rh-catalyzed asymmetric hydrogenation using $\mathrm{L}_{9}-\mathrm{L}_{17}{ }^{a}$

\begin{tabular}{|c|c|c|c|c|}
\hline Entry & Ligand & $\begin{array}{l}\text { Substrate I } \\
\mathrm{ee}^{b}\end{array}$ & $\begin{array}{l}\text { Substrate II } \\
\mathrm{ee}^{b}\end{array}$ & $\begin{array}{l}\text { Substrate III } \\
\mathrm{ee}^{b}\end{array}$ \\
\hline 1 & L9 & $96(R)$ & $91(R)$ & $98(R)$ \\
\hline 2 & L10 & $90(R)$ & $67(R)$ & $66(R)$ \\
\hline 3 & L11 & $41(S)$ & $64(S)$ & $91(S)$ \\
\hline 4 & L12 & $95(R)$ & $40(R)$ & $97(R)$ \\
\hline 5 & L13 & $93(R)$ & $87(R)$ & $92(R)$ \\
\hline 6 & L14 & $88(R)$ & $76(R)$ & $79(R)$ \\
\hline 7 & L15 & $95(R)$ & $92(R)$ & $94(R)$ \\
\hline 8 & L16 & $46(R)$ & $2(R)$ & $15(R)$ \\
\hline $9^{c}$ & L17 & $97(R)$ & - & $96(R)$ \\
\hline
\end{tabular}

${ }^{a}$ Reaction conditions: In a stainless steel autoclave, Rh/substrate $=$ $1: 30, p\left(\mathrm{H}_{2}\right)=1.2 \mathrm{bar}, T=25^{\circ} \mathrm{C}, t=16 \mathrm{~h}, 0.5 \mathrm{~mL}$ of THF, all runs were performed in duplicate, full conversion was obtained in all cases, conversion was determined by GC. ${ }^{b}$ Enantiomeric excess of product determined by chiral GC (absolute configuration drawn in parenthesis). ${ }^{c}$ Data taken from ref. 18. in this case changing $R^{1}$ from a phenyl group to a cyclohexyl group led to the opposite enantiomer with varying levels of selectivity for all three of the tested substrates. This nicely showcases that small changes in ligand structure can have a huge influence on the outcome of a catalytic reaction and demonstrates the power of this effective modular approach towards the synthesis and screening of large supported P-OP ligand libraries.

Lastly different phosphite moieties and their influence on the selectivity were investigated. Both a slightly more bulky $\mathrm{SiMe}_{3}$ substituted $(S)$-binol and an achiral $t$-butyl substituted bisphenol derived phosphite were employed. From Table 2 it can be concluded that changing to a $\mathrm{SiMe}_{3}$ substituted binol moiety ( $\mathbf{L}_{\mathbf{1 3}}$ and $\mathbf{L}_{\mathbf{1 4}}$, entries 5 and 6) in most cases has a small but positive effect on the selectivity when compared to their non-substituted binol counterparts $\mathbf{L}_{\mathbf{2}}$ and $\mathbf{L}_{\mathbf{6}}$ (Table 1 , entries 2 and 6). Going to the achiral $t$-butyl bisphenol phosphite did seem to have a large influence resulting in lower enantioselectivities. For $\mathbf{L}_{\mathbf{1 5}}$ with a C3 ligand backbone (Table 2, entry 7 ) enantioselectivities up to $11 \%$ lower than for the binol bearing counterpart $\mathbf{L}_{2}$ (Table 1 , entry 2) were observed. In the case of $\mathbf{L}_{\mathbf{1 6}}$ bearing a $\mathbf{C} 4$ backbone (Table 2, entry 8 ) the effect of changing to the achiral bisphenol moiety was much larger. For $\mathbf{L}_{\mathbf{1 6}}$ a significant drop in selectivity of respectively $48 \%$ and $80 \%$ was found for substrates I and III when compared to the parent ligand $\mathbf{L}_{\mathbf{6}}$ (Table 1 , entry 6). Moreover for substrate II surprisingly, a complete loss of selectivity was observed.

When compared to phosphine-phosphite $\mathbf{L}_{\mathbf{1 7}}$ reported by Bakos et al. (Table 2, entry 9), ${ }^{18}$ essentially the homogeneous counterpart of resin-bound ligand $\mathbf{L}_{2}$, it was found that some of the supported ligands performed very well. For substrate I it can be seen that comparable enantioselectivities were obtained (Table 1, entry 9) and for substrate III supported P-OP ligand $\mathbf{L}_{\mathbf{9}}$ even outperforms it solution-phase analogue by $2 \%$ (Table 2, entry 1). This is quite remarkable as in most known cases the immobilization of a homogeneous catalyst has a detrimental effect on the selectivity. This shows that the modular solid-phase synthetic approach demonstrated here not only enables the facile synthesis and screening of large P-OP ligand libraries but can also actually lead to highly selective supported catalysts.

\section{Supported catalysis recycling}

To assess the reusability of these supported phosphine-phosphites the best performing member of the ligand library $\mathbf{L}_{\mathbf{9}}$ was employed in the asymmetric hydrogenation of acetamidocinnamic acid methyl ester III. The catalyst recycling was performed under batch conditions in a Schlenk vessel under a flow of hydrogen. In between each reaction cycle the resin was washed with substrate solution while maintaining a hydrogen atmosphere to ensure catalyst stability. Finally the reaction time was reduced in the recycling experiments to study recycling at low conversion, which gives a better indication of the actual catalyst stability. 


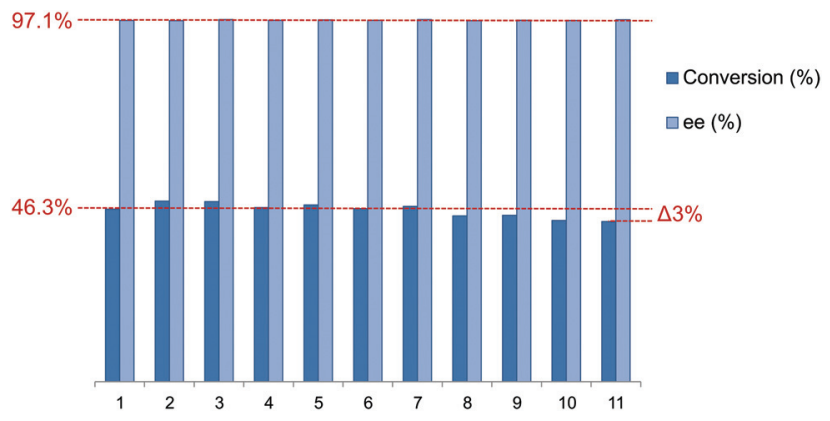

Fig. 6 Results of catalyst recycling of supported ligand $L_{9}$ in the Rhcatalyzed asymmetric hydrogenation of substrate III. Reaction conditions: In a Schlenk vessel under $\mathrm{H}_{2}$ atmosphere, $\mathrm{Rh} /$ substrate $=1: 30$, $p\left(\mathrm{H}_{2}\right)=1 \mathrm{~atm}, T=25^{\circ} \mathrm{C}, t=20 \mathrm{~min}, 1.5 \mathrm{~mL}$ of THF, all runs were performed in duplicate.

The results of the catalyst recycling experiments are summarized in Fig. 6. Merrifield supported ligand $\mathbf{L}_{\mathbf{9}}$ could be reused up to 7 times without any loss of activity. After 11 runs only a very marginal drop in conversion of $3.3 \%$ was observed. The loss of activity might have been caused by the possible introduction of trace amounts of moisture or air during workup in between reaction cycles. Moreover the selectivity stayed constant (average 97.2\%) over the full extent of the recycling experiments.

Also the metal leaching into solution after each reaction cycle was analyzed using ICP-OES. After the first reaction cycle an initial Rh leaching of $1.6 \mathrm{ppm}$ was found and the metal leaching appeared to stay constant after 3 recycling runs and only a minimal leaching of on average $1.3 \mathrm{ppm}$ was detected (for full results see ESI, Table S-I†). The fact that no initial decrease in catalytic activity was observed despite that some metal leaching was detected, seems to indicate the leaching might actually be caused by physically adsorbed rhodium residues residing in the pores of the resin. Moreover no phosphorus leaching could be observed after analyzing the reaction solution with ${ }^{31} \mathrm{P}$ NMR which also supports that the small decrease in catalytic activity is probably caused by other deactivation processes than leaching of active complex into solution. As phosphite-based ligands are prone to hydrolysis and phosphines to oxidation $\mathbf{L}_{\mathbf{g}}$ shows remarkable stability for a phosphine-phosphite. ${ }^{25}$ Potentially this type of supported P-OP ligands could provide promising candidates for catalysis under flow conditions.

\section{Conclusion}

A new modular solid-phase synthetic procedure allowing the facile synthesis of large and diverse libraries of phosphinephosphites was developed. Using this approach a library of 16 phosphine-phosphite ligands could be obtained in very high yields. Moreover only a very minimal work-up was required between reaction steps as opposed to the laborious purification methods employed in the solution-phase synthesis of similar ligands, often resulting in low yields. The supported ligand library was successfully employed in Rh-catalyzed asymmetric hydrogenation and three benchmark substrates were screened. Very high enantioselectivities up to $98 \%$ were achieved. Moreover some members of the ligand library even outperformed a homogeneous counterpart showing that the immobilization did not have a detrimental effect on the selectivity. Finally supported ligand $\mathbf{L}_{\mathbf{9}}$ was employed in catalyst recycling under batch conditions. The ligand, immobilized on Merrifield resin, could be successfully reused for 11 reaction cycles showing only a very minor loss of activity. Moreover, no decrease in selectivity was observed throughout the recycling experiments. The exhibited recyclability is remarkably high for these types of ligands and might make this system highly suitable for catalysis under flow conditions.

\section{Experimental section}

\section{General experimental}

All reactions and manipulations were carried out under inert conditions using standard Schlenk techniques or in an MBraun glovebox unless stated otherwise. All glassware was dried prior to use to remove traces of water. All chemicals were obtained from commercial suppliers and used as received unless otherwise stated. Diethyl ether and THF were distilled from sodium/benzophenone and triethylamine, dichloromethane and acetonitrile were distilled from calcium hydride. Novabiochem $^{\mathrm{TM}}$ Merrifield resin (100-200 mesh, $1.24 \mathrm{mmol}$ $\mathrm{g}^{-1}$ or $1.48 \mathrm{mmol} \mathrm{g}^{-1}, 1 \%$ cross-linked) was obtained from EMD Millipore.

\section{General procedure for the synthesis of resin-bound phosphine-boranes $\left(\mathbf{1 a}-\mathbf{b} \cdot \mathrm{BH}_{3}\right)$}

Step 1. Merrifield resin $\left(2.0 \mathrm{~g}, 1.24 \mathrm{mmol} \mathrm{g}^{-1}, 2.48 \mathrm{mmol}, 1.0\right.$ eq.), was swollen in THF $(50 \mathrm{~mL})$ and cooled to $-78{ }^{\circ} \mathrm{C}$. A freshly prepared primary lithium phosphide solution $(20 \mathrm{~mL}$, $0.15 \mathrm{M}, 1.2$ eq.), also cooled to $-78^{\circ} \mathrm{C}$ was added under gentle stirring to avoid mechanical abrasion of the resin. The reaction mixture was allowed to warm up to room temperature and was left overnight without stirring. The supernatant was removed and the resin was washed subsequently with three $20 \mathrm{~mL}$ portions of THF followed by three $20 \mathrm{~mL}$ portions of $\mathrm{Et}_{2} \mathrm{O}$. The product was directly used in the next step without additional purification.

Step 2. A resin-bound phosphine, synthesized in the previous step, was swollen in THF $(50 \mathrm{~mL})$. Next, $\mathrm{BH}_{3} \cdot \mathrm{SMe}_{2}$ $(12.5 \mathrm{~mL}, 2.0 \mathrm{M}$ in toluene, $10 \mathrm{eq}$.) was added under gentle stirring to avoid mechanical abrasion of the resin. Upon addition the resin colored white and the reaction was stopped when full conversion was observed by ${ }^{31} \mathrm{P}$ NMR. Next, the supernatant was removed and the resin was washed subsequently with three $20 \mathrm{~mL}$ portions of THF followed by three $20 \mathrm{~mL}$ of $\mathrm{Et}_{2} \mathrm{O}$. The product was dried in vacuo yielding a white resin-bound phosphine-borane. 
General procedure for the synthesis of resin-bound phosphine-borane sulfates $\left(3 \mathrm{a}-\mathrm{h} \cdot \mathrm{BH}_{3}\right)$

Step 1. A resin-bound phosphine-borane $\left(\mathbf{1 a}-\mathbf{b} \cdot \mathbf{B H} \mathbf{H}_{3}, 500 \mathrm{mg}\right.$, $\sim 0.6 \mathrm{mmol})$ was swollen in THF $(20 \mathrm{~mL})$. Next, LDA $(3 \mathrm{~mL}$, 2.0 $\mathrm{M}$ in THF/heptane/ethylbenzene, $10 \mathrm{eq}$.) was added under gentle stirring to avoid mechanical abrasion of the resin. Upon addition the resin colored dark brown and was allowed to react for 3 hours. Next, the supernatant was removed and the resin was washed subsequently with three $10 \mathrm{~mL}$ portions of THF followed by three $10 \mathrm{~mL}$ portions of $\mathrm{Et}_{2} \mathrm{O}$. The product was used in the next step without additional purification.

Step 2. A lithiated resin-bound phosphine-borane synthesized in the previous step was swollen in THF $(15 \mathrm{~mL})$. A cyclic sulfate (2a-e, $0.72 \mathrm{mmol}, 1.2 \mathrm{eq}$.) was azeotropically dried with toluene ( 3 times), dissolved in THF ( $5 \mathrm{~mL}$ ) and subsequently added to the resin under gentle stirring to avoid mechanical abrasion. Upon addition the resin turned from dark brown to yellow and was allowed to react overnight. Next, the supernatant was removed and the resin was washed subsequently with three $10 \mathrm{~mL}$ portions of THF and three $10 \mathrm{~mL}$ portions of $\mathrm{Et}_{2} \mathrm{O}$. The product was dried in vacuo yielding a light yellow resin. The product was used in the next step without additional purification.

General procedure for the synthesis of resin-bound hydroxyalkyl phosphine-boranes $\left(\mathbf{4 a}-\mathrm{h} \cdot \mathrm{BH}_{3}\right)$

A resin-bound phosphine-borane sulfate $\left(\mathbf{3 a}-\mathbf{h} \cdot \mathbf{B H} \mathbf{H}_{3}, 500 \mathrm{mg}\right.$, $\sim 0.5 \mathrm{mmol}$ ) was swollen, under gentle stirring, in a 1:1 mixture of THF and $0.1 \mathrm{M}$ of degassed $\mathrm{H}_{2} \mathrm{SO}_{4}(20 \mathrm{~mL})$. The resin was left overnight without stirring to avoid mechanical abrasion of the resin. Next the resin was washed with three $5 \mathrm{~mL}$ portions of THF and resuspended in THF $(10 \mathrm{~mL})$. The progress of the hydrolysis was monitored using ${ }^{7} \mathrm{Li} \mathrm{NMR}$. If no full consumption of $\mathrm{Li}$ was observed the resin was resuspended in a fresh mixture of THF and $\mathrm{H}_{2} \mathrm{SO}_{4}$ and left overnight. This procedure was repeated until no lithium signal could be observed anymore by ${ }^{7} \mathrm{Li}$ NMR (on average 3 days). Next, the supernatant was removed and the resin was washed subsequently with five $10 \mathrm{~mL}$ portions of THF followed by three $10 \mathrm{~mL}$ portions of $\mathrm{Et}_{2} \mathrm{O}$. The product was dried in vacuo affording a white resin which was used in the next step without additional purification.

\section{General procedure for the synthesis of resin-bound phosphine-phosphites $\left(\mathbf{L}_{1}-\mathbf{L}_{16}\right)$}

Step 1. A resin-bound hydroxyalkyl phosphine-borane (4a$\left.\mathbf{h} \cdot \mathbf{B H}_{3}, 250 \mathrm{mg}, \sim 0.25 \mathrm{mmol}\right)$ was suspended in THF (5 mL) and a solution of 1,4-diazabicyclo[2.2.2] octane (5 mL, 0.5 $\mathrm{M}$ in THF, 10 eq.) was added. The reaction was heated to $40^{\circ} \mathrm{C}$ and was left overnight without stirring. After complete deprotection was confirmed by ${ }^{31} \mathrm{P} \mathrm{NMR}$, the supernatant was removed and the resin was washed subsequently with three $5 \mathrm{~mL}$ portions of THF followed by three $5 \mathrm{~mL}$ portions of $\mathrm{Et}_{2} \mathrm{O}$. The product was used directly in the next step without further purification.
Step 2. A deprotected resin-bound hydroxyalkyl phosphine synthesized in the previous step was swollen in THF $(10 \mathrm{~mL})$ and triethylamine $(2.25 \mathrm{mmol}, 9.0$ eq.) was added. A chlorophosphite (0.75 mmol, 3.0 eq.) was dissolved in THF $(5 \mathrm{~mL})$ and was added to the resin at $0{ }^{\circ} \mathrm{C}$ under gentle stirring to avoid mechanical abrasion of the resin. Upon addition a precipitate was formed. The reaction was monitored using ${ }^{31} \mathrm{P}$ NMR and full conversion was reached when a 1:1 ratio of phosphine to phosphite was observed (2-16 hours). Next, the supernatant was removed and the resin was washed subsequently with three $5 \mathrm{~mL}$ portions of DCM, three $5 \mathrm{~mL}$ portions of THF and three $5 \mathrm{~mL}$ portions of $\mathrm{Et}_{2} \mathrm{O}$. The product was dried in vacuo yielding a white resin-bound phosphinephosphite.

\section{General procedure for Rh-catalyzed asymmetric hydrogenation}

The hydrogenation experiments were performed in a stainless steel autoclave charged with an insert suitable for 10 reaction vessels including Teflon mini stirring bars. In a typical experiment, a reaction vessel was charged with a resin-bound phosphine-phosphite $(5 \mathrm{mg}, \sim 4.0 \mu \mathrm{mol})$ and a solution of [Rh$\left.(\mathrm{COD})_{2}\right] \mathrm{X}$ (0.9 eq.) in $\mathrm{CH}_{2} \mathrm{Cl}_{2}(1 \mathrm{~mL})$ was added and the heterogeneous mixture was allowed to stir gently for $4 \mathrm{~h}$. The supernatant was removed and the resulting orange resin was washed subsequently with three $1 \mathrm{~mL}$ portions of THF followed by three $1 \mathrm{~mL}$ portions of $\mathrm{Et}_{2} \mathrm{O}$. Next, a solution of substrate $(0.5 \mathrm{~mL}, 0.24 \mathrm{M}, 30 \mathrm{eq}$.) in THF was added to the reaction vessel. Subsequently, the autoclave was purged three times with 5 bar of $\mathrm{H}_{2}$ and then pressurized to 1.2 bar. The reaction mixtures were gently stirred at $25{ }^{\circ} \mathrm{C}$. After $16 \mathrm{~h}$, the autoclave was depressurized and the reaction mixtures were filtered over a plug of silica. Prior to GC measurements substrate II and its products were derivatized using (trimethylsilyl)diazomethane ( $2 \mathrm{M}$ in diethyl ether), in essence yielding substrate III. The conversion and the enantiomeric excess were determined by chiral GC, see ESI $\uparrow$ for column and conditions.

\section{Acknowledgements}

We thank the European Union (Marie Curie ITN SusPhos, grant agreement no. 317404) for financial support.

\section{Notes and references}

1 (a) H. U. Blaser and E. Schmidt, Asymmetric Catalysis on Industrial Scale, ed. H. U. Blaser and E. Schmidt, WileyVCH Verlag GmbH \& Co. KGaA, Weinheim, 2004; (b) I. Ojima, Catalytic asymmetric synthesis, John Wiley \& Sons, Inc., Hoboken, 3rd edn, 2010.

2 T. P. Yoon and E. N. Jacobsen, Science, 2003, 299, 1691.

3 (a) K. N. Houk and P. H.-Y. Cheong, Nature, 2008, 455, 309; (b) M. C. Kozlowski and M. Panda, J. Org. Chem., 2003, 68, 2061. 
4 (a) M. B. Francis, N. S. Finney and E. N. Jacobsen, J. Am. Chem. Soc., 1996, 118, 8983; (b) R. Kranich, K. Eis, O. Geis, S. Mühle, J. W. Bats and H.-G. Schmalz, Chem. - Eur. J., 2000, 6, 2874; (c) C. Gennari and U. Piarulli, Chem. Rev., 2003, 103, 3071.

5 (a) M. T. Reetz, Angew. Chem., Int. Ed., 2001, 40, 284; (b) J. G. de Vries and A. H. M. de Vries, Eur. J. Org. Chem., 2003, 799.

6 (a) P. E. Goudriaan, X.-B. Jang, M. Kuil, R. Lemmens, P. W. N. M. van Leeuwen and J. N. H. Reek, Eur. J. Org. Chem., 2008, 6079; (b) B. Breit, Angew. Chem., Int. Ed., 2005, 44, 6816; (c) G. Hattori, T. Hori, Y. Miyake and Y. Nishibayashi, J. Am. Chem. Soc., 2007, 129, 12930.

7 (a) L. Lefort, J. A. F. Boogers, A. H. M. de Vries and J. G. de Vries, Org. Lett., 2004, 6, 1733; (b) M. T. Reetz and G. Mehler, Tetrahedron Lett., 2003, 44, 4593; (c) M. L. Johansson, S. Berglund, Y. Hu, K. H. O. Andersson and N. Kann, ACS Comb. Sci., 2012, 14, 304.

8 P. E. Goudriaan, P. W. N. M. van Leeuwen, M. N. Birkholz and J. N. H. Reek, Eur. J. Inorg. Chem., 2008, 2939.

9 (a) S. Lühr, J. Holz and A. Börner, ChemCatChem, 2011, 3, 1708; (b) S. H. Chikkali, J. I. van der Vlugt and J. N. H. Reek, Coord. Chem. Rev., 2014, 262, 1; (c) H. Fernandez-Perez, P. Etayo, A. Panossian and A. VidalFerran, Chem. Rev., 2011, 111, 2119.

10 N. Sakai, S. Mano, K. Nozaki and H. Takaya, J. Am. Chem. Soc., 1993, 115, 7033.

11 (a) A. Suárez, M. A. Méndez-Rojas and A. Pizzano, Organometallics, 2002, 21, 4611; (b) P. W. N. M. van Leeuwen, P. C. J. Kamer, C. Claver, O. Pamies and M. Dieguez, Chem. Rev., 2011, 111, 2077.

12 (a) C. F. Czauderna, D. B. Cordes, A. M. Z. Slawin, C. Müller, J. I. van der Vlugt, D. Vogt and P. C. J. Kamer, Eur. J. Inorg. Chem., 2014, 1797; (b) M. Dindaroğlu, A. Falk and H.-G. Schmalz, Synthesis, 2013, 527; (c) H. FernándezPérez, M. A. Pericàs and A. Vidal-Ferran, Adv. Synth. Catal., 2008, 350, 1984; (d) J. L. Núñez-Rico, P. Etayo, H. Fernández-Pérez and A. Vidal-Ferran, Adv. Synth. Catal., 2012, 354, 3025; (e) J. Velder, T. Robert, I. Weidner, J.-M. Neudörfl, J. Lex and H.-G. Schmalz, Adv. Synth. Catal., 2008, 350, 1309; (f) O. Pàmies, M. Diéguez, G. Net, A. Ruiz and C. Claver, J. Org. Chem., 2001, 66, 8364.

13 (a) S. E. Booth, C. M. Dreef-Tromp, P. H. H. Hermkens, J. A. P. A. de Man and H. C. J. Ottenheijm, in Combinatorial Chemistry - Synthesis, Analysis, Screening, ed. G. Jung, WileyVCH Verlag GmbH, Weinheim, 1999, pp. 35-76; (b) D. Obrecht and J. M. Villalgordo, in Solid-Supported Combinatorial and Parallel Synthesis of Small-MolecularWeight Compound Libraries, Elsevier Science ltd, Oxford, 1998, pp. 1-184; (c) R. B. Merrifield, J. Am. Chem. Soc., 1963, 85, 2149.

14 M. C. Samuels, B. H. G. Swennenhuis and P. C. J. Kamer, in Phosphorus(III) Ligands in Homogeneous Catalysis: Design and Synthesis, ed. P. C. J. Kamer and P. W. N. M. van Leeuwen, John Wiley \& Sons, Ltd, 2012, pp. 463-479.

15 (a) J. N. H. Reek, P. W. N. M. van Leeuwen, A. G. J. Ham and A. B. Haan, in Catalyst Separation, Recovery and Recycling, ed. D. J. Cole-Hamilton and R. P. Tooze, Springer, Netherlands, 2006, pp. 39-72; (b) T. E. Kristensen and T. Hansen, in Catalytic Methods in Asymmetric Synthesis, ed. M. Gruttadauria and F. Giacalone, John Wiley \& Sons, Inc., 2011, pp. 209-256; (c) G. Zhao and Z. Chai, in Recoverable and Recyclable Catalysts, ed. M. Benaglia, John Wiley \& Sons, Ltd, 2009, pp. 49-75; (d) A. F. Trindade, P. M. P. Gois and C. A. M. Afonso, Chem. Rev., 2009, 109, 418.

16 (a) G. Y. Li, P. J. Fagan and P. L. Watson, Angew. Chem., Int. Ed., 2001, 113, 1140; (b) R. den Heeten, B. H. G. Swennenhuis, P. W. N. M. van Leeuwen, J. G. de Vries and P. C. J. Kamer, Angew. Chem., Int. Ed., 2008, 47, 6602; (c) A. Mansour and M. Portnoy, J. Chem. Soc., Perkin Trans. 1, 2001, 952; (d) B. Bar-Nir Ben-Aroya and M. Portnoy, Tetrahedron, 2002, 58, 5147; (e) A. Mansour and M. Portnoy, Tetrahedron Lett., 2003, 44, 2195.

17 F. J. L. Heutz, M. C. Samuels and P. C. J. Kamer, Catal. Sci. Technol., 2015, 5, 3296.

18 G. Farkas, S. Balogh, Á. Szöllősy, L. Ürge, F. Darvas and J. Bakos, Tetrahedron: Asymmetry, 2011, 22, 2104.

19 (a) A. Panossian, H. Fernández-Pérez, D. Popa and A. VidalFerran, Tetrahedron: Asymmetry, 2010, 21, 2281; (b) C. Hegedüs, H. Gulyás, Á. Szöllősy and J. Bakos, Inorg. Chim. Acta, 2009, 362, 1650.

20 G. Fries, J. Wolf, K. Ilg, B. Walfort, D. Stalke and H. Werner, Dalton Trans., 2004, 1873.

21 P. W. N. M. van Leeuwen and J. C. Chadwick, in Homogeneous Catalysts: Activity - Stability - Deactivation, WileyVCH Verlag GmbH \& Co. KGaA, 2011, pp. 1-49.

22 (a) S. Deerenberg, P. C. J. Kamer and P. W. N. M. van Leeuwen, Organometallics, 2000, 19, 2065; (b) S. Deerenberg, H. S. Schrekker, G. P. F. van Strijdonck, P. C. J. Kamer, P. W. N. M. van Leeuwen, J. Fraanje and K. Goubitz, J. Org. Chem., 2000, 65, 4810.

23 T. T. Adint and C. R. Landis, J. Am. Chem. Soc., 2014, 136, 7943.

24 (a) Z. Hua, V. C. Vassar and I. Ojima, Org. Lett., 2003, 5, 3831; (b) K. N. Gavrilov, S. V. Zheglov, P. A. Vologzhanin, E. A. Rastorguev, A. A. Shiryaev, M. G. Maksimova, S. E. Lyubimov, E. B. Benetsky, A. S. Safronov, P. V. Petrovskii, V. A. Davankov, B. Schäffner and A. Börner, Russ. Chem. Bull., 2008, 57, 2311.

25 (a) R. B. Bedford, S. J. Coles, M. B. Hursthouse and V. J. Scordia, Dalton Trans., 2005, 991; (b) R. Chen, J. Jiang, Y. Wang and Z. Jin, J. Mol. Catal. A: Chem., 1999, 149, 113; (c) B. H. G. Swennenhuis, R. Chen, P. W. N. M. van Leeuwen, J. G. de Vries and P. C. J. Kamer, Eur. J. Org. Chem., 2009, 5796. 